\title{
Perturbed Infinite 3D Simple Cubic Network of Identical Capacitors
}

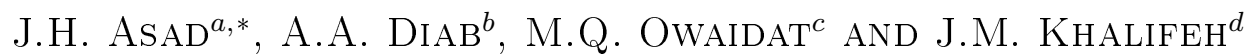 \\ ${ }^{a}$ Department of Physics, College of Arts and Sciences, Palestine Technical University, P.O. Box 7, Tulkarm, Palestine \\ ${ }^{b}$ Department of General Studies, Yanbu Industrial College, P.O. Box 30436, Yanbu Industrial City, Saudi Arabia \\ ${ }^{c}$ Department of Physics, Al-Hussein Bin Talal University, Ma'an 71111, Jordan \\ ${ }^{d}$ Department of Physics, The University of Jordan, Amman 11942, Jordan
}

(Received March 31, 2014; in final form July 24, 2014)

\begin{abstract}
The effective capacitance between the origin and any other lattice site, in an infinite 3D simple cubic network consisting of identical capacitors, is evaluated in terms of the lattice Green function of the network. The perfect case is reviewed shortly, while the perturbed case (a capacitor is removed) is studied in two cases. Numerical values of the effective capacitance are presented and the asymptotic behavior is studied for the both cases.
\end{abstract}

DOI: 10.12693 /APhysPolA.126.777

PACS: 61.50.Ah, 84.37.+q, 05.50.+q, 04.60.Nc, 63.90.+t, 84.30.Bv, 02.90.+p

\section{Introduction}

Green function theory has a great importance in physics and mathematics. It is strongly used in many areas of physics such as diffusion [1], band structure [2, 3] lattice dynamics [4-6] and statistical models of ferromagnetism like Ising model [7], Heisenberg model [8], spherical model [9] and random walks [10-13]. One of the most common applications of Green's function theory is the lattice Green function (LGF), which is widely used as a basic function in condensed matter. The LGF for cubic lattices has been investigated by many authors [14-22], and the so-called recurrence formulae are obtained to calculate LGF for simple cubic (SC), body centered cubic (BCC) and face centered cubic (FCC) lattices [17, 18]. Numerical values of LGF for these lattices have been recently exactly evaluated and expressed rationally in terms of the known value of the LGF at the origin [22].

In recent years, the LGF is successfully applied to calculate resistance in many different infinite networks of resistors $[23,24]$. The computation of the resistance between two nodes in infinite and finite resistor networks is a classic problem in electric circuit theory which is of interests to physicist as well as to electrical engineering is [25-42]. This problem was studied by many authors, and one can see that many methods have been used.

A similar problem of the same interests to physicists and electrical engineering in the electric circuit theory is the calculation of effective capacitance in infinite networks. Less attention has been paid on to study this problem [43-46]. In this work the LGF method is used to study both the perfect infinite and perturbed SC network consisting of identical capacitors. The LGF of the SC lattice used in this work is related to the Green function of

\footnotetext{
* corresponding jasad@ptuk.edu.ps

author;

e-mail: drjasad@yahoo.com,
}

the tight-binding Hamiltonian (TBH) [12]. Effective capacitance of perturbed SC network has been expressed in terms of effective capacitance of perfect case.

The present work is organized as follows: in Sect. 2 a brief revision is carried out for the perfect infinite $\mathrm{SC}$ network. In Sect. 3 an application is carried out to calculate effective capacitance. This section is divided into two parts. In part 1 we revised shortly the perfect infinite SC network consisting of identical capacitors, while in part 2 we studied the perturbed SC network due to removing a capacitor. We close this paper with analysis and discussion to the results obtained.

Finally, we believe that the method presented here is educational and of great importance for undergraduate physicists and engineering students.

\section{Basic definitions and preliminaries}

In this section, we will introduce some basic preliminaries for the perfect infinite SC network. First of all, we assume that all lattice points are specified by the following position vector:

$$
\boldsymbol{r}=n_{1} \boldsymbol{a}_{1}+n_{2} \boldsymbol{a}_{2}+n_{3} \boldsymbol{a}_{3},
$$

where $n_{1}, n_{2}, n_{3}$ are integers (positive, negative or zero), and $\boldsymbol{a}_{1}, \boldsymbol{a}_{2}, \boldsymbol{a}_{3}$ are independent primitive transition vectors with equal magnitudes.

For such perfect infinite SC network, the LGF was expressed rationally as [22]:

$$
G_{0}\left(3 ; n_{1}, n_{2}, n_{3}\right)=r_{1} g_{0}+\frac{r_{2}}{\pi^{2} g_{0}}+r_{3},
$$

where $g_{0}$ is the LGF of the perfect infinite SC lattice at the origin. It was evaluated in the Watson classic paper [11], where he showed that $g_{0}=G_{0}(0,0,0)=$ $\left(\frac{2}{\pi}\right)^{2}(18+12 \sqrt{2}-10 \sqrt{3}-7 \sqrt{6})\left[K\left(k_{0}\right)\right]^{2}=0.505462$. $k_{0}=(2-\sqrt{3})(\sqrt{3}-\sqrt{2})$, and $K(k)=\int_{0}^{\frac{\pi}{2}} \mathrm{~d} \theta \frac{1}{\sqrt{1-k^{2} \sin ^{2} \theta}}$ 
is the complete elliptic integral of the first kind, while $r_{1}, r_{2}$ and $r_{3}$ are rational numbers related to Duffin and Shelly's parameter as [47]:

$$
r_{1}=\lambda_{1}+\frac{15}{12} \lambda_{2} ; \quad r_{2}=-\frac{1}{2} \lambda_{2} ; \quad r_{3}=\frac{1}{3} \lambda_{3} .
$$

Various values of $r_{1}, r_{2}$ and $r_{3}$ are presented in Ref. [22]. Other values can be calculated using the following recurrence relation:

$$
\begin{aligned}
& G_{0}(E ; l+1, m, n)+G_{0}(E ; l-1, m, n) \\
& \quad+G_{0}(E ; l, m+1, n)+G_{0}(E ; l, m-1, n) \\
& \quad+G_{0}(E ; l, m, n+1)+G_{0}(E ; l, m, n-1)= \\
& \quad-2 \delta_{l 0} \delta_{m 0} \delta_{n 0}+2 E G_{0}(E ; l, m, n),
\end{aligned}
$$

where $E=3$ is the energy of the infinite SC lattice at the band.

The asymptotic behavior of the LGF $G_{0}\left(3 ; n_{1}, n_{2}, n_{3}\right)$ is interesting to our study. One can show that as any of $n_{1}, n_{2}, n_{3}$ goes to infinity, then [23] $G_{0}\left(3 ; n_{1}, n_{2}, n_{3}\right) \rightarrow 0$.

\section{Application: calculation of effective capacitance in $\mathrm{SC}$ network}

\subsection{Perfect case}

Now, consider that the perfect infinite SC network, discussed in Sect. 2 above, consists of identical capacitors each of capacitance $C$. Figure 1 shows a section of the infinite network of identical capacitors. The dark spheres

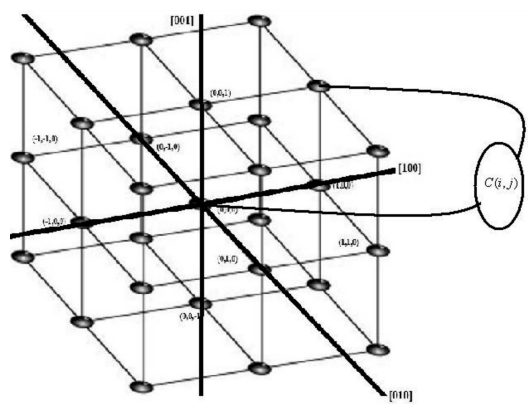

Fig. 1. A section of the infinite network of identical capacitors. The dark spheres represent the lattice sites.

represent the lattice sites. Our aim is finding the effective capacitance between the origin of the network and any other site $\left(n_{1}, n_{2}, n_{3}\right)$. It has been shown that [46]:

$$
C\left(n_{1}, n_{2}, n_{3}\right)=\frac{C}{\left[G_{0}(3 ; 0,0,0)-G_{0}\left(3 ; n_{1}, n_{2}, n_{3}\right)\right]} .
$$

Making use of Eq. (2) the effective capacitance can be expressed as [48]:

$$
C\left(n_{1}, n_{2}, n_{3}\right)=\frac{C}{\left[\sigma_{1} g_{0}+\frac{\sigma_{2}}{\pi^{2} g_{0}}+\sigma_{3}\right]} .
$$

Here $\sigma_{1}, \sigma_{2}, \sigma_{3}$ are rational numbers related to those defined in Eq. (3) as:

$$
\sigma_{1}=1-r_{1}=1-\lambda_{1}-\frac{15}{12} \lambda_{2}
$$

$$
\sigma_{2}=-r_{2}=\frac{1}{2} \lambda_{2} ; \quad \sigma_{3}=-r_{3}=\frac{1}{3} \lambda_{3} .
$$

Various values of $\sigma_{1}, \sigma_{2}, \sigma_{3}$ (and, of course, various values of effective capacitance) can be found in [48]. In this paper we quoted some of them in Tables I and II below for comparison reason.

To study the asymptotic behavior of the effective capacitance as the separation between the origin and the site $\left(n_{1}, n_{2}, n_{3}\right)$ goes to infinity we simply insert the fact that $G_{0}\left(3 ; n_{1}, n_{2}, n_{3}\right) \rightarrow 0$ into Eq. (5) where we got

$$
C\left(n_{1}, n_{2}, n_{3}\right)=\frac{C}{G_{0}(3 ; 0,0,0)} \rightarrow \text { finite value. }
$$

\subsection{Perturbed case}

In this section, we consider a perturbed case in which it is a perfect infinite SC network but with removing the capacitor between sites $i_{0}$ and $j_{0}$. Our target is finding the effective capacitance between the sites $i=\left(i_{x}, i_{y}, i_{z}\right)$ and $j=\left(j_{x}, j_{y}, j_{z}\right)$ for the perturbed case. To do this, we first define the charge contribution $\delta Q_{i}$ at the site $\boldsymbol{r}_{i}$ due to the bond $\left(i_{0} j_{0}\right)$ is given by:

$$
\begin{aligned}
& \frac{\delta Q_{i}}{C}=\delta_{i i_{0}}\left(V_{i_{0}}-V_{j_{0}}\right)+\delta_{i j_{0}}\left(V_{j_{0}}-V_{i_{0}}\right)= \\
& \left\langle i \mid i_{0}\right\rangle\left(\left\langle i_{0}\right|-\left\langle j_{0}\right|\right) V+\left\langle i \mid j_{0}\right\rangle\left(\left\langle j_{0}\right|-\left\langle i_{0}\right|\right) V= \\
& \left\langlei \left|\left(\left|i_{0}\right\rangle-\left|j_{0}\right\rangle\right)\left(\left\langle i_{0}\right|-\left\langle j_{0}\right|\right) V=\langle i| L_{1} V,\right.\right.
\end{aligned}
$$

where the operator $L_{1}$ has the form

$$
L_{1}=\left(\left|i_{0}\right\rangle-\left|j_{0}\right\rangle\right)\left(\left\langle i_{0}\right|-\left\langle j_{0}\right)\right. \text {. }
$$

Now, removing the bond $\left(i_{0} j_{0}\right)$ from the perfect lattice the charge $Q_{i}$ at the site $\boldsymbol{r}_{i}$ is given as

$$
\left(-L_{0} V\right)_{i}-\frac{1}{C} \delta Q_{i}=\frac{-Q_{i}}{C} .
$$

Ohm's and Kirchhoff's laws for the perturbed lattice may be written as

$$
L_{01} V=\frac{-Q}{C},
$$

where

$$
L_{01}=L_{0}+L_{1} \text {. }
$$

Similarly to the perfect lattice, the LGF $G_{01}$ for the perturbed lattice is defined as:

$$
L_{01} G_{01}=-1 \text {. }
$$

Therefore, Eq. (12) becomes

$$
V=\frac{G_{01} Q}{C} \text {. }
$$

Here the operator $L_{01}$ is now a sum of $L_{0}$ associated with the perfect lattice and a perturbation given by $L_{1}$.

To calculate the capacitance between the sites $\boldsymbol{r}_{i}$ and $\boldsymbol{r}_{j}$, we assume the charge to be given as

$$
Q_{m}=Q\left[\delta_{m i}-\delta_{m j}\right] \text { for all } m \text {. }
$$

As a result Eq. (15) becomes

$$
\begin{aligned}
& V_{k}=\langle k| V=\frac{\langle k| G_{01} Q}{C}=\frac{1}{C} \sum_{m}\left\langle k\left|G_{01}\right| m\right\rangle Q_{m}= \\
& \frac{Q}{C}\left[G_{01}(k, i)-G_{01}(k, j)\right] .
\end{aligned}
$$


Thus, the capacitance between the lattice sites $\boldsymbol{r}_{i}$ and $\boldsymbol{r}_{j}$ is

$$
\begin{aligned}
& C_{01}(i, j)= \\
& \quad \frac{C}{\left[G_{01}(i, i)-G_{01}(i, j)+G_{01}(j, j)-G_{01}(j, i)\right]} .
\end{aligned}
$$

Note here that $G_{01}(i, i) \neq G_{01}(j, j)$ since the translational symmetry is broken, but $G_{01}(i, j)=G_{01}(j, i)$. Our problem of finding the capacitances reduces to the calculation of the perturbed LGF, because once we calculate it then from Eq. (18) we find the perturbed capacitances on the networks.

Instead of the above idea, in the following we write the perturbed capacitance of the network in terms of the perfect ones.

Using the following equation:

$$
G_{01}=G_{0}+G_{0} L_{1} G_{0}+G_{0} L_{1} G_{0} L_{1} G_{0}+\ldots
$$

To solve the above formula one can use the method presented by Economou [12]. Inserting Eq. (10) into the above equation, one gets

$$
\begin{aligned}
& G_{01}(i, j)=\left\langle i\left|G_{01}\right| j\right\rangle=G_{0}(i, j) \\
& \quad+\frac{\left[G_{0}\left(i, i_{0}\right)-G_{0}\left(i, j_{0}\right)\right]\left[G_{0}\left(i_{0}, j\right)-G_{0}\left(j_{0}, j\right)\right]}{1-2\left[G_{0}\left(i_{0}, i_{0}\right)-G_{0}\left(i_{0}, j_{0}\right)\right]} .
\end{aligned}
$$

Finally, the capacitance between $\boldsymbol{r}_{i}$ and $\boldsymbol{r}_{j}$ can be obtained in terms of the perfect capacitances and can be written after some simply straightforward algebra as

$$
\begin{aligned}
& \frac{C_{01}(i, j)}{C}= \\
& \frac{1}{C_{0}(i, j)}+\frac{\left.1 \frac{1}{C_{0}\left(i, j_{0}\right)}+\frac{1}{C_{0}\left(j, i_{0}\right)}-\frac{1}{C_{0}\left(i, i_{0}\right)}-\frac{1}{C_{0}\left(j, j_{0}\right)}\right]^{2}}{4\left[1-\frac{1}{C_{0}\left(i_{0}, j_{0}\right)}\right]}
\end{aligned}
$$

This is our final result for the perturbed capacitance between the sites $\boldsymbol{r}_{i}$ and $\boldsymbol{r}_{j}$ in which the bond $\left(i_{0} j_{0}\right)$ is removed. Remember here that our case (i.e., SC lattice) $i=\left(i_{x}, i_{y}, i_{z}\right)$ and $j=\left(j_{x}, j_{y}, j_{z}\right)$.

It is important to study the asymptotic behavior of the capacitance as the separation between $i$ and $j$ goes to infinity. In this case the above equation becomes

$$
\frac{C_{01}(i, j)}{C} \rightarrow \frac{1}{\frac{C}{C_{0}(i, j)}}=\frac{C_{0}(i, j)}{C} \rightarrow \frac{1}{g_{0}} .
$$

This means that the perturbed capacitance (i.e. $\left.C_{01}(i, j)\right)$ approaches to a perfect capacitance (i.e. $\left.C_{0}(i, j)\right)$. This result means that a large separation between the sites $\boldsymbol{r}_{i}$ and $\boldsymbol{r}_{j}$ will not be affected by removing a capacitor between sites $\boldsymbol{r}_{i_{0}}$ and $\boldsymbol{r}_{j_{0}}$.

\section{Numerical results and discussion}

In this section, numerical results are presented for an infinite SC network consisting of identical capacitors including the perfect and both of the perturbed cases. The capacitance between the sites $i=(0,0,0)$ and $j=\left(j_{x}, j_{y}, j_{z}\right)$ in an infinite perfect SC lattice is calculated in Asad et al. [48]. Some calculated values are quoted in Tables I and II below for comparison reasons.

For the case of the perturbed network, one has to specify exactly the two ends of the removed bond and then
TABLE I

Calculated values for the effective capacitance of an infinite SC lattice between the sites $i=(0,0,0)$ and $j=$ $\left(j_{x}, j_{y}, j_{z}\right)$, for a perfect lattice $\left(C_{0}(i, j) / C\right.$; perturbed lattice due to removing a capacitor between $(0,0,0)$, $(1,0,0)$ and $(1,0,0),(2,0,0):\left(C_{1}(i, j) / C\right)$ and $\left(C_{2}(i, j) / C\right)$, respectively, along the direction [100].

\begin{tabular}{c|l|l|l}
\hline \hline Site & $\frac{C_{1}(i, j)}{C}$ & $\frac{C_{2}(i, j)}{C}$ & $\frac{C_{0}(i, j)}{C}$ \\
\hline$(0,0,0)$ & $\infty$ & $\infty$ & $\infty$ \\
$(1,0,0)$ & 2 & 2.80734375 & 3 \\
$(2,0,0)$ & 2.058741 & 2.058741087 & 2.38275 \\
$(3,0,0)$ & 1.999744508 & 2.166582332 & 2.22039 \\
$(4,0,0)$ & 1.95978919 & 2.127560921 & 2.15107 \\
$(5,0,0)$ & 1.934045382 & 2.096091576 & 2.113000 \\
$(6,0,0)$ & 1.916446256 & 2.074195321 & 2.08885 \\
$(-1,0,0)$ & 2.80734375 & 2.989579852 & 3 \\
$(-2,0,0)$ & 2.202492142 & 2.371811714 & 2.38275 \\
$(-3,0,0)$ & 2.047127764 & 2.209203787 & 2.22039 \\
$(-4,0,0)$ & 1.982225632 & 2.139782845 & 2.15107 \\
$(-5,0,0)$ & 1.947155124 & 2.101677630 & 2.113000 \\
$(-6,0,0)$ & 1.925157797 & 2.070409258 & 2.08885
\end{tabular}

TABLE II

Calculated values for the effective capacitance of an infinite SC lattice between the sites $i=(0,0,0)$ and $j=$ $\left(j_{x}, j_{y}, j_{z}\right)$, for a perfect lattice $\left(C_{0}(i, j) / C\right.$; perturbed lattice due to removing a capacitor between $(0,0,0)$, $(1,0,0)$ and $(1,0,0),(2,0,0):\left(C_{3}(i, j) / C\right)$ and $\left(C_{4}(i, j) / C\right)$, respectively, along the direction [111].

\begin{tabular}{c|l|l|l}
\hline \hline Site & $\frac{C_{3}(i, j)}{C}$ & $\frac{C_{4}(i, j)}{C}$ & $\frac{C_{0}(i, j)}{C}$ \\
\hline$(0,0,0)$ & $\infty$ & $\infty$ & $\infty$ \\
$(1,1,1)$ & 2.146010787 & 2.38208889 & 2.3906 \\
$(2,2,2)$ & 1.98571039 & 2.158864628 & 2.17316 \\
$(3,3,3)$ & 1.93232867 & 2.092081721 & 2.10516 \\
$(4,4,4)$ & 1.905828163 & 2.059833427 & 2.07224 \\
$(5,5,5)$ & 1.890032967 & 2.040839542 & 2.05286 \\
$(6,6,6)$ & 1.879496211 & 2.028280023 & 2.04006 \\
$(-1,-1,-1)$ & 2.200860052 & 2.379997988 & 2.3906 \\
$(-2,-2,-2)$ & 1.999637226 & 2.161759091 & 2.17316 \\
$(-3,-3,-3)$ & 1.938444258 & 2.093667729 & 2.10516 \\
$(-4,-4,-4)$ & 1.909231269 & 2.060778636 & 2.07224 \\
$(-5,-5,-5)$ & 1.89211771 & 2.041455849 & 2.05286 \\
$(-6,-6,-6)$ & 1.880955737 & 2.028719701 & 2.040067
\end{tabular}

the values of the perturbed capacitance can be calculated using the calculated values of the perfect SC lattice (i.e. $\left.C_{0}(i, j)\right)$ and Eq. (13).

In this work we considered two cases.

First, the bond between $i_{0}=(0,0,0)$ and $j_{0}=(1,0,0)$ is removed.

Second, then the removed bond is shifted and set between $i_{0}=(1,0,0)$ and $j_{0}=(2,0,0)$.

We calculated the effective capacitance between the sites $i=(0,0,0)$ and $j=\left(j_{x}, j_{y}, j_{z}\right)$ along the directions [100] and [111] for the above two perturbed cases. Some calculated values are presented in Tables I and II below. 
In Figs. 2-3, the effective capacitance for the perfect and the above two perturbed cases are plotted as a function of the site along [100] direction, while in Figs. 4-5, the effective capacitance for the perfect and the above two perturbed cases are plotted as a function of the site along [111] direction. From these figures, we can see that the effective capacitance in the perfect network is always greater than that in the perturbed case, and this is obvious from Eq. (13).

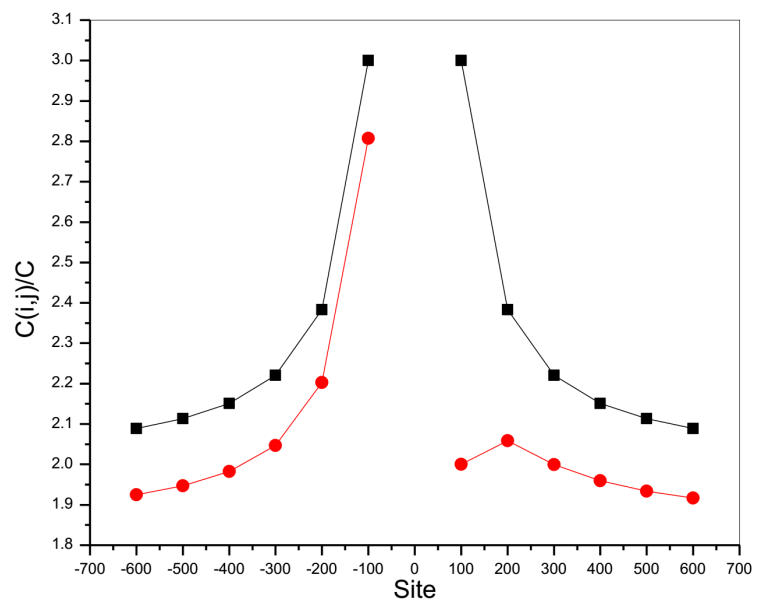

Fig. 2. The capacitance between $i=(0,0,0)$ and $j=$ $\left(j_{x}, 0,0\right)$ along [100] direction of the perfect (squares) and the perturbed infinite SC lattice (circles) as a function of $j_{x}$. The ends of the removed capacitor are $i_{0}=(0,0,0)$ and $j_{0}=(1,0,0)$.

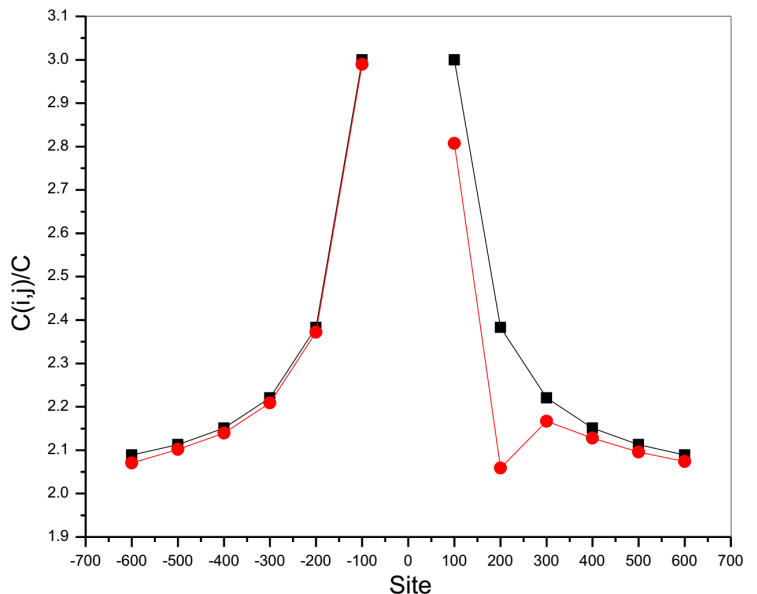

Fig. 3. The capacitance between $i=(0,0,0)$ and $j=$ $\left(j_{x}, 0,0\right)$ along [100] direction of the perfect (squares) and the perturbed infinite SC lattice (circles) as a function of $j_{x}$. The ends of the removed capacitor are shifted and become between $i_{0}=(1,0,0)$ and $j_{0}=(2,0,0)$.

Finally, from Figs. $2-5$ it is clear that the effective capacitance in the infinite perfect $\mathrm{SC}$ network is symmetric. But for the two perturbed cases the effective capacitance

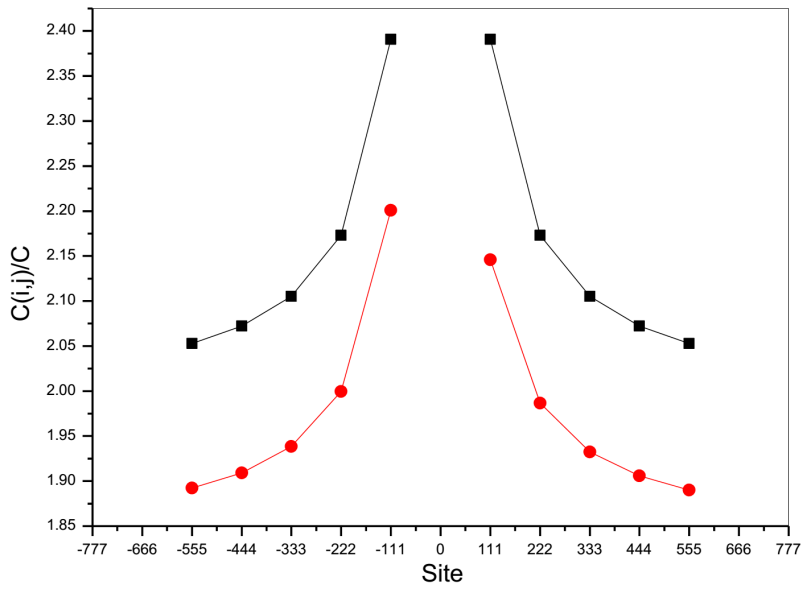

Fig. 4. The capacitance between $i=(0,0,0)$ and $j=$ $\left(j_{x}, j_{y}, j_{z}\right)$ along [111] direction of the perfect (squares) and the perturbed infinite SC lattice (circles) as a function of the site $j=\left(j_{x}, j_{y}, j_{z}\right)$. The ends of the removed capacitor are $i_{0}=(0,0,0)$ and $j_{0}=(1,0,0)$.

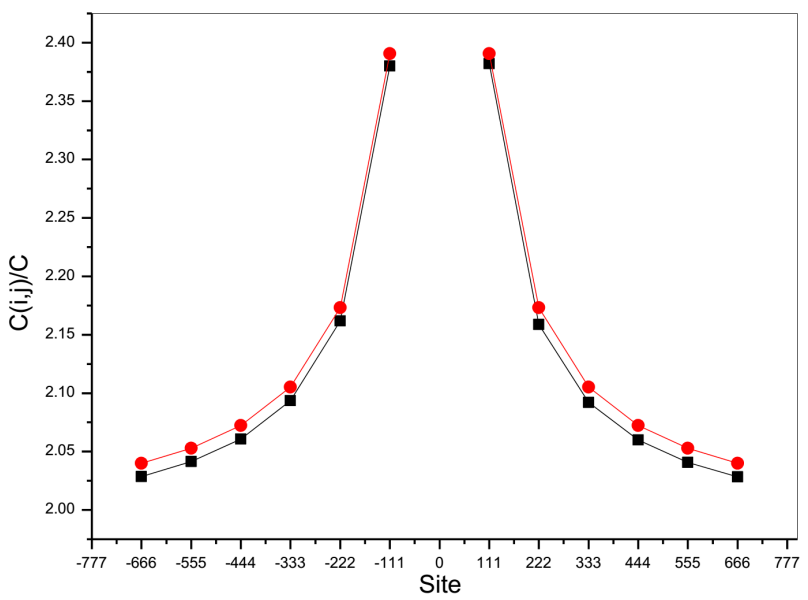

Fig. 5. The capacitance between $i=(0,0,0)$ and $j=$ $\left(j_{x}, j_{y}, j_{z}\right)$ along [111] direction of the perfect (squares) and the perturbed infinite SC lattice (circles) as a function of the site $j=\left(j_{x}, j_{y}, j_{z}\right)$. The ends of the removed capacitor are shifted and become between $i_{0}=(1,0,0)$ and $j_{0}=(2,0,0)$.

is not symmetric due to the fact that the inversion symmetry of the infinite lattice has been broken.

\section{References}

[1] G.L. Montent, Phys. Rev. B 7, 650 (1975).

[2] G.F. Koster, D.C. Slater, Phys. Rev. 96, 1208 (1954).

[3] V. Bryksin, P. Kleinert, Z. Phys. B, Condens. Matter 90, 167 (1993).

[4] P.H. Dederichs, K. Shroeder, R. Zeller, Point Defect in Metals II, Springer-Verlag, Berlin 1980.

[5] B. Hughes, J. Math. Phys. 23, 1688 (1982). 
[6] G.F. Koster, J.C. Slater, Phys. Rev. 94, 1498 (1954).

[7] N.W. Dalton, D.W. Wood, Proc. Phys. Soc. (London) 90, 4591 (1967).

[8] M. Lax, Phys. Rev. 97, 629 (1955).

[9] E. Montroll, G. Weiss, J. Math. Phys. 6, 168 (1965).

[10] E.W. Montroll, R.B. Potts, Phys. Rev. 100, 525 (1955).

[11] G.N. Watson, Quart. J. Math (Oxford) 10, 266 (1939).

[12] E.N. Economou, Green's Functions in Quantum Physics, 2nd ed., Springer-Verlag, Berlin 1983.

[13] R. Brout, Phys. Rev. 118, 1009 (1960).

[14] G.S. Joyce, J. Math. Phys. 12, 1390 (1971).

[15] S. Katsura, T. Horiguchi, J. Math. Phys. 12, 230 (1971).

[16] T. Morita, T. Horiguci, Math. Phys. 12, 986 (1971).

[17] T. Horiguchi, J. Phys. Soc. Jpn. 30, 1261 (1971).

[18] M.L. Glasser, J. Math. Phys. 13, 1145 (1972).

[19] K. Mano, J. Math. Phys. 16, 1726 (1975).

[20] M. Inoue, J. Math. Phys. 16, 809 (1975).

[21] T. Morita, T. Horiguchi, J. Phys. C, Solid State Phys. 8, L232 (1975).

[22] M.L. Glasser, J. Boersma, J. Phys. A, Math. Gen. 33, 5017 (2000).

[23] J. Cserti, Am. J. Phys. 68, 896 (2000).

[24] J. Cserti, D. Gyula, P. Attila, Am. J. Phys. 70, 153 (2002).

[25] M. Jeng, Am. J. Phys. 68, 37 (2000).

[26] R.E. Aitchison, Am. J. Phys. 32, 566 (1964).

[27] G. Venezian, 10.1119/1.17696 Am. J. Phys. 62, 1000 (1994).

[28] D. Atkinson, F.J. van Steenwijk, Am. J. Phys. 67, 486 (1999).

[29] J.H. Asad, R.S. Hijjawi, A. Sakaji, J.M. Khaleh, Int. J. Theor. Phys. 43, 2223 (2004).
[30] J.H. Asad, R.S. Hijjawi, A. Sakaji, J.M. Khaleh, Int. J. Theor. Phys. 44, 471 (2004).

[31] J.H. Asad, A.J. Sakaji, R.S. Hijjawi, J.M. Khalifeh, Eur. Phys. J. B 52, 365 (2006).

[32] R.S. Hijjawi, J.H. Asad, A.J. Sakaji, M. Al-Sabayleh, J.M. Khalifeh, EPJ Appl. Phys. 41, 111 (2008).

[33] J. Cserti, G. Szechenyi, G. David, J. Phys. A, Math. Gen. 44, 215201 (2011).

[34] F.Y. Wu, J. Phys. A, Math. Gen. 37, 6653-6673 (2004).

[35] J.W. Essam, F.Y. Wu, J. Phys. A, Math. Theor. 42, 025205 (2009)

[36] N.Sh. Izmailian, M.-C. Huang, Phys. Rev. E 82, 011125 (2010).

[37] J.H. Asad, A.A. Diab, R.S. Hijjawi, J.M. Khalifeh, Eur. Phys. J. Plus 128, 1 (2013).

[38] J.H. Asad, J. Stat. Phys. 150, 1177 (2013).

[39] M.Q. Owaidat, R.S. Hijjawi, J.M. Khalifeh, Mod. Phys. Lett. B 19, 2057 (2010).

[40] M.Q. Owaidat, R.S. Hijjawi, J.M. Khalifeh, J. Phys. A, Math. Theor. 43, 375204 (2010).

[41] M.Q. Owaidat, R.S. Hijjawi, J.M. Khalifeh, Int. J. Theor. Phys. 51, 3152 (2012).

[42] J.H. Asad, R.S. Hijjawi, A.J. Sakaji, J.M. Khalifeh, Int. J. Mod. Phys. B 19, 3713 (2005).

[43] R.S. Hijjawi, J.H. Asad, A.J. Sakaji, J.M. Khalifeh, Int. J. Mod. Phys. B 21, 199 (2007).

[44] J.H. Asad, A.J. Sakaji, R.S. Hijjawi, J.M. Khalifeh, Eur. Phys. J. Appl. Phys. 40, 257 (2007).

[46] J.H. Asad, R.S. Hijjawi, A.J. Sakaji, J.M. Khalifeh, Eur. Phys. J. Appl. Phys. 32, 149 (2005).

[47] R.J. Duffin, E.P. Shelly, Duke Math. J. 25, 209 (1958).

[48] J.H. Asad, Mod. Phys. Lett. B. 27, 15 (2013). 